\title{
Influência da extensão universitária nos atributos da atenção primária a saúde no contexto da puericultura
}

\author{
Influence of the university extension on the attributes of primary health care in the context of child care \\ Influencia de la ampliación universitaria sobre los atributos de la atención primaria de salud en el contexto de la \\ atención infantil \\ Luana Bartsch 1 (D) https://orcid.org/0000-0001-7488-8311 \\ Maiara Florencia Loronha ${ }^{1}$ (D) https://orcid.org/0000-0002-8508-4324 \\ Nathalia Piazentini Pioczkoski ${ }^{1}$ (D) https://orcid.org/0000-0002-0941-2197 \\ Veronica Souza Cavalheiro ${ }^{1}$ (D) https://orcid.org/0000-0002-9244-181x \\ Andrea Moreira Arrue ${ }^{2}$ (D) https://orcid.org/0000-0001-5391-324X \\ Leonardo Bigolin Jantsch ${ }^{1}$ (D) https://orcid.org/0000-0002-4571-183x
}

\section{Resumo}

Objetivo: Avaliar a influência das atividades de extensão universitária nos atributos da Atenção Primária em Saúde (APS) no contexto da puericultura.

Métodos: Trata-se de estudo quase experimental com grupo controle não equivalente, que analisou os escores dos atributos em dois cenários: grupo Intervenção (cenário que participou de projeto de extensão em puericultura), e o grupo controle (demais cenários da APS). 0 instrumento de avaliação (PCATOOL, versão criança) foi aplicado a cuidadores de lactentes, que acessaram a APS. A análise dos atributos foi por comparação de média, entres os grupos analisados (teste t).

Resultados: Os resultados mostram que o grupo intervenção, que recebeu as ações extensionistas, obteve melhor orientação à APS $(p=0,01)$ bem como seguiu com práticas de aleitamento materno mais efetivas $(p>0,01)$ comparado ao grupo controle. 0 atributo prestação de serviço foi melhor, no grupo intervenção, quando comparado ao controle $(p<0,05)$.

Conclusão: Incentivar atividades extensionistas universitárias em puericultura contribuem para a melhoria na avaliação da APS e nos desfechos de aleitamento materno.

\section{Abstract}

Objective: To evaluate the influence of university extension activities on the attributes of Primary Health Care (PHC) in the context of childcare.

Methods: This is a quasi-experimental study with a non-equivalent control group, which analyzed the attribute scores in two scenarios: Intervention group (scenario that participated in an extension project in childcare), and the control group (other PHC scenarios). The assessment instrument (PCATOOL, child version) was applied to caregivers of infants who accessed the PHC. Attributes were analyzed by comparing the means between the analyzed groups ( $t$ test).

Results: The results show that the intervention group, which received the extension actions, obtained better guidance on PHC ( $p=0.01)$ and followed with more effective breastfeeding practices $(p>0.01)$ compared to the control group. The service provision attribute was better, in the intervention group, when compared to the control $(p<0.05)$. Conclusion: Encouraging university extension activities in childcare contribute to improving the assessment of PHC and breastfeeding outcomes.

\section{Resumen}

Objetivo: Evaluar la influencia de las actividades de extensión universitaria sobre los atributos de la Atención Primaria de Salud (APS) en el contexto del cuidado infantil.

Metodos: Se trata de un estudio cuasiexperimental con un grupo de control no equivalente, que analizó las puntuaciones de los atributos en dos escenarios: grupo de intervención (escenario que participó en un proyecto de extensión en cuidado infantil) y grupo de control (otros escenarios de APS). El instrumento de evaluación (PCATOOL, versión infantil) se aplicó a los cuidadores de los lactantes que accedían a la APS. Los atributos se analizaron comparando las medias entre los grupos analizados (prueba t).

Resultados: Los resultados muestran que el grupo de intervención, que recibió las acciones de extensión, obtuvo una mejor orientación en APS $(p=0.01)$ y siguió con prácticas de lactancia materna más efectivas $(p>0.01)$ en comparación con el grupo control. El atributo de prestación de servicios fue mejor, en el grupo de intervención, en comparación con el control $(p<0,05)$.

Conclusión: Fomentar las actividades de extensión universitaria en el cuidado infantil contribuye a mejorar la evaluación de los resultados de la APS y la lactancia materna.

\section{Como citar:}

Bartsch L, Loronha MF, Pioczkoski NP, Cavalheiro VS, Arrue AM, Jantsch LB. Influência da extensão universitária nos atributos da atenção primária a saúde no contexto da puericultura. Rev Soc Bras Enferm Ped. 2021;21(2):166-72.

\section{Descritores}

Cuidado da criança; Atenção primária à saúde; Pesquisa sobre serviços de saúde; Relações comunidade-instituição

\section{Keywords}

Child care; Primary health care; Research on health services; Communityinstitutional relations

Universidade Federal de Santa Maria, Palmeira das Missões, RS, Brasil.

${ }^{2}$ Universidade Federal do Paraná, Curitiba, PR, Brasil.

Conflitos de interesse: nada a declarar.

Submetido: 10 de Setembro de 2021 | Aceito: 15 de Dezembro de 2021

Autor correspondente: Leonardo Bigolin Jantsch | E-mail: leonardo.jantsch@ufsm.br

Dol: http://dx.doi.org/10.31508/1676-379320210023 


\section{Introdução}

A Atenção Primária à Saúde (APS) é definida como o conjunto de ações em saúde, coletivas ou individuais, que compreendem a promoção e proteção da saúde, prevenção de doenças, diagnóstico e tratamento, redução de danos e manutenção das condições de saúde da população. ${ }^{(1)}$ Nessa perspectiva, a população pode ter acesso aos serviços de saúde, no âmbito da APS, por meio das equipes de Estratégia Saúde da Família (ESF), considerado a principal estratégia de acolhimento e vinculação da comunidade.

É no cenário da APS, especialmente nas ESF, que as ações de atenção à saúde da criança são organizadas e reconhecidas como Puericultura. A palavra Puericultura vem do latim "puerus" que significa criança, sendo são consideradas as ações de promoção à saúde da criança, desenvolvidas durante as consultas realizadas pelo enfermeiro ou médico na ESF. Estas ações têm como objetivo incentivar o aleitamento materno, acompanhar o crescimento e desenvolvimento da criança, orientar quanto a higiene e introdução alimentar e a prevenção de acidentes, acompanhar a carteira de vacinação, dentre outras ações que ajudam na construção de vínculos e reforçam a importância do acompanhamento regular. A equipe de saúde precisa estar integrada para prestar um atendimento qualificado, coerente com as necessidades das crianças e suas famílias. O enfermeiro tem um papel importante como coordenador da equipe e colaborador para a implantação de estratégias que melhore o acompanhamento de saúde das crianças. ${ }^{(2)}$

As estratégias governamentais brasileiras recomendam que as consultas de puericultura devam seguir o seguinte esquema de acompanhamento, quan-

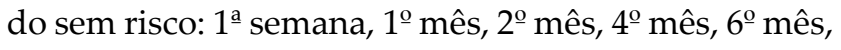
$9^{\circ}$ mês, $12^{\circ}$ mês, $18^{\circ}$ mês e $24^{\circ}$ mês, a partir dos dois anos de idade a consulta deve ser anual e próxima ao mês do aniversário. Nesta primeira consulta do recém-nascido ( $1^{\circ}$ semana de vida), é o momento propício parar a equipe estimular e auxiliar a família nas dificuldades do aleitamento materno exclusivo, orientar sobre as imunizações, verificar o teste do pezinho, a caderneta de saúde da criança, identificação de riscos e vulnerabilidade, bem como estabelecer uma rede de apoio à família. É essencial a sequência nas consultas de Puericultura proporcionando o melhor crescimento, a partir da promoção e prevenção de agravos. ${ }^{(3)}$
A ESF é um espaço de atuação que oferece muitas potencialidades de aprendizados para os acadêmicos da área da saúde, especialmente no que se refere a extensão universitária. A aproximação do ensino, dos serviços de saúde e da comunidade pode trazer benefícios na organização e prestação de serviços, na nas experiências e formação dos acadêmicos, na melhoria dos vínculos com a comunidade e famílias. ${ }^{(4)}$

De acordo com a Política de Extensão Universitária, as atividades de extensão realizadas pelas instituições de ensino, devem seguir algumas diretrizes para orientação e formulação das atividades, são elas: Interação Dialógica, Interdisciplinaridade e Interprofissionalidade, Indissociabilidade Ensino-Pesquisa Extensão, Impacto na Formação do Estudante, e Impacto e Transformação Social. ${ }^{(5)}$

A extensão universitária na APS, além de melhorar a orientação à APS, permite o engajamento e autonomia dos estudantes, para o desenvolvimento nas experiências teórico-práticas, especialmente na oportunidade de integrar as questões do ensino, serviço e comunidade. Cabe destacar que trata-se de uma ferramenta importante no desenvolvimento do aluno, que acompanha uma realidade diferente daquela vista na sala de aula. Caracteriza uma colaboração positiva para a comunidade, em que o estudante socializa e compartilha saberes podendo conhecer a realidade profissional. Espera-se então com essas atividades o amadurecimento do acadêmico, como a formação de raciocínio crítico e crescimento profissional. ${ }^{(6)}$

No campo da saúde, as avaliações são consideradas importantes estratégias para mensurar a qualidade dos serviços prestados, as fragilidades e as potencialidades dos processos de trabalho. Ela acontece por meio de instrumentos já consolidados e validados mundialmente. ${ }^{(7)}$ No âmbito da saúde da criança e as avaliações dos serviços da APS ainda são poucos $^{(8)}$ e por isso, a necessidade de avaliar a realização das atividades de puericultura em duas ESF, destacando o impacto das ações de extensão na avaliação da APS, sobretudo na perspectiva dos usuários dos serviços de saúde. Além da incipiente presença de estudos que avaliam a orientação da APS no contexto da puericultura, são inexistentes estudos que apontam estratégias e/ou avaliação das atividades de puericultura realizadas por instituições de ensino superior. 
Nessa direção, de hipótese inicial, reconhece-se que as atividades de extensão em puericultura melhoram as avaliações da APS, especialmente nos atributos essenciais que são: o acesso ao primeiro contato, longitudinalidade, integralidade e coordenação da atenção bem como prestação de serviços. Para tanto o presente estudo tem por objetivo: Avaliar a influência das atividades de extensão universitária nos atributos da Atenção Primária em Saúde no contexto da Puericultura.

\section{Métodos}

Trata-se de um estudo analítico, quase-experimental com grupo controle não equivalente, que comparou os escores dos atributos de avaliação da APS em cenários da puericultura com e sem intervenção de atividades de extensão. Serão comparados dois cenários, um denominado de "intervenção" onde foram realizadas atividades de extensão universitária, em consultas de puericultura e, outro grupo, denominado "controle" considerados as demais ESF do município que não foram contempladas com as atividades do projeto.

O município em estudo localiza-se na região noroeste do Rio Grande do Sul e faz parte dos municípios da 15 ${ }^{\text {a }}$ Coordenadoria Regional de Saúde (CRS). Possui área territorial de 1.421,101 km², população estimada de 33.303 pessoas, densidade demográfica de 24,18 hab/ $\mathrm{km}^{2}$, e mortalidade infantil de 9,51 óbitos por mil nascidos vivos. ${ }^{(9)}$ No município com cobertura de 100\% de Estratégia de Saúde da Família (ESF), possui 10 unidades de ESF, distribuídas em nove urbanas e uma rural mais um ambulatório, com atendimento de especialidades médicas de referência para o município (ginecologia e obstetrícia, pediatria).

As atividades de extensão em puericultura são realizadas desde janeiro de 2019 e integram o Projeto intitulado "Consulta em Puericultura: contribuições para a saúde, serviço e formação", desenvolvido em parceria com duas ESF. As atividades realizadas no projeto baseiam-se em consultas de enfermagem, estabelecidas por roteiro e orientações descritas no Caderno no 33 da Atenção Básica. Os atendimentos incluíam, prioritariamente crianças de zero a dois anos e são realizados mediante agendamento de demanda específica e busca ativa. Foram realizadas de seis a oito consultas por turno, uma vez por semana, totalizando em torno de 300 consultas em um ano de execução do projeto. As consultas foram realizadas por acadêmicos de enfermagem, sendo um deles bolsista do projeto, acompanhados integralmente por professor orientador (enfermeiro especialista em enfermagem pediátrica e neonatal).

Os dados foram coletados nos meses de outubro de 2019 a fevereiro de 2020, encerrando as coletas presenciais nas ESF, com o início da pandmeia do COVID-19. Participaram da pesquisa, familiares (considerados pelos participantes) de lactentes (recém-nascidos até 1 ano, 11 meses e 29 dias de vida) que tivessem mais de 18 anos e que o lactente fosse residente na área de abrangência da ESF em que se estava coletando os dados. Ainda, a criança precisaria ter utilizado o serviço no mínimo em dois momentos, podendo ser consulta e/ou procedimento, com exceção da vacinação exclusiva. E como critério de exclusão, familiares que desconhecem das condições de saúde de lactentes, não estando aptos para responder as perguntas.

Foi realizado um cálculo amostral simples representativo partindo da população de 1008 lactentes do município, ${ }^{(9)}$ utilizando o nível de confiança de $95 \%$ e margem de erro de 5\%, estabelecendo uma amostra de 279 lactentes. Em decorrência a pandemia de Covid-19 no Brasil, houve a suspensão das atividades extensionistas e, desta forma, foram coletados 75 instrumentos em todas as dez ESF, sendo 38 instrumentos do Grupo Intervenção e 37 instrumentos do Grupo Controle. Essa nova projeção considerou uma amostra populacional simples, com nível de confiança de $95 \%$ e um erro de $9 \%$.

A equipe de coleta selecionou, de forma presencial em visita a campo (nas dez ESF urbanas do município local do estudo), por meio de demanda espontânea, usuários que procurassem a ESF para consulta, realização de procedimentos, vacina, entre outras demandas da APS e estivessem aguardando por esse serviço na sala de espera da unidade de saúde. O familiar/ cuidador selecionado, aleatoriamente, confirmava se possui alguma criança menor de dois anos na família e se conhece as condições de saúde para responder os questionamentos do instrumento.

Inicialmente foi realizada uma capacitação sobre a coleta de dados para a equipe de coletadores (acadêmicos de enfermagem), com a finalidade de conhecer e explorar o instrumento de pesquisa. Para coleta dos 
dados, forma utilizados instrumentos: Instrumento de Caracterização, referentes ao perfil socioeconômico e algumas variáveis como os antecedentes obstétricos e neonatais, Instrumento de Classificação Socioeconômica com dados sobre avaliação socioeconômica e moradia da criança, avaliação da APS (PCAToolCriança) e avaliação da Condição Crônica desde o nascimento até o período atual.

Foram consideradas variáveis desfecho o escore de avaliação dos atributos da APS avaliação [escore (como variável numérica), Alta Orientação ou Baixa orientação (como variável categórica)] da atenção primária a saúde, condições de saúde, aleitamento materno, internações hospitalares e seguimento na puericultura. Foi considerada variável exposição a distribuição no grupo intervenção e grupo controle (dicotomia).

O instrumento Avaliação da Atenção Primária a Saúde (PCATool Criança) baseia-se na mensuração de aspectos de estrutura, processo e resultados dos serviços de saúde.(1) O instrumento é composto por 55 itens divididos em dez componentes relacionados aos atributos da APS, os escores que apresentam valores de zero a quatro. Nesse estudo foi utilizado a versão atualizada PCATool Brasil/2020(10) na avaliação dos atributos essenciais e derivados, de acordo com as orientações da referência adotada. ${ }^{(10)} \mathrm{O}$ Manual sugere classificar em Alto $(\geq 6,6)$ onde há presença e extensão dos atributos, mostrando serviços mais bem orientados pela APS, e Baixo $(\leq 6,6)$, esse valor do escore em 6,6 reflete as respostas aos itens do instrumento com no mínimo a categoria de resposta 'provavelmente sim', atribuído ao código três na escala original, portanto, minimamente presentes as características de serviços em saúde reconhecidos como orientados à APS.

Os dados coletados em instrumento físico, foram digitados em planilhas Excel, sob dupla digitação e conferência. Foram analisados os dados por meio da comparação entre os dois grupos, consideramos Grupo controle (participantes que pertenciam a ESF que não recebera as ações de extensão) e grupo intervenção (participantes que pertenciam a ESF que recebera as atividades de extensão propostas). As análises foram realizadas por meio do programa SPSS Statistics, versão 20.0, utilizando o Teste Qui-quadrado ou Teste exato de Fisher para comparação de frequência entre dois grupos (variáveis categóricas), Razão de Risco (RR), nas variáveis dicotômicas e o teste $t$ para amostras inde- pendentes, tendo em vista normalidade dos dados (verificada pelo teste de Shapiro-Wilk), para comparar a média entre os dois grupos (para variáveis numéricas). Estabeleceu-se nível de significância de 5\%, para significância estatística tendo em vista um confiança de 95\%.

O estudo seguiu as recomendações da Resolução 466/2012, foi aprovado pelo comitê de ética em pesquisa, sob Número do Parecer: 3.425.473 com data de aprovação em 07/05/2019.

\section{Resultados}

De acordo com a tabela 1, podemos notar que o grupo controle e o grupo intervenção, assim denominados, possuem características semelhantes referentes à idade gestacional e peso ao nascer e idade no momento da avaliação, sem diferença entre as médias nos grupos.

Tabela 1. Média e desvio padrão da idade gestacional, peso ao nascer e idade dos lactentes participantes do estudo

\begin{tabular}{|c|c|c|c|}
\hline Características & $\begin{array}{c}\text { Grupo } \\
\text { Intervenção } \\
\text { Média(Desvio- } \\
\text { Padrão) }\end{array}$ & $\begin{array}{l}\text { Grupo Controle } \\
\text { Média(Desvio- } \\
\text { Padrão) }\end{array}$ & $p$-value* \\
\hline Idade gestacional & 38,4 & 38,2 & 0,740 \\
\hline Peso ao nascer (gramas) & $3051(386)$ & $3039(400)$ & 0,890 \\
\hline Idade do lactente (meses) & $8,42(5,3)$ & $6,23(5,4)$ & 0,08 \\
\hline
\end{tabular}

Na comparação das variáveis relacionadas a condições de saúde, socioeconômicas e de acompanhamento em puericultura, descreve-se a tabela 2 .

De acordo com os dados da tabela 2, observou-se diferença estatística entre os grupos nas variáveis adesão ao aleitamento materno e aleitamento materno exclusivo. Mulheres do grupo intervenção apresentaram maior adesão ao aleitamento materno e a permanecer com esta condição de forma exclusiva em comparação às do grupo controle. Demais condições avaliadas não foram diferentes entre os grupos comparados. Quanto a avaliação geral dos atributos da APS, conforme tabela 3, não foi verificada diferença estatisticamente entre os grupos intervenção e controle.

Na tabela 4 observa-se que o escore médio da avaliação geral e do atributo $\mathrm{H}$, foram maiores no grupo intervenção em comparação ao controle $(p<0,05)$. Quando avaliados isoladamente os demais atributos da APS não foram significantes do ponto de vista estatístico. 
Tabela 2. Condições de Saúde e acompanhamento de puericultura dos lactentes, participantes do estudo, estratificados por grupo analisado

\begin{tabular}{|c|c|c|c|c|}
\hline Variáveis & $\begin{array}{c}\text { Grupo Intervenção } \\
\mathrm{n}(\%)\end{array}$ & $\begin{array}{c}\text { Grupo Controle } \\
n(\%)\end{array}$ & p-valor & $* * * \operatorname{RR}(\mathrm{IC} 95 \%)$ \\
\hline \multicolumn{5}{|l|}{ Condições de saúde } \\
\hline Possui Condição Crônica de Saúde & $2(5,3)$ & $1(3,7)$ & $0,572 *$ & $1,50(0,29-7,55)$ \\
\hline Hospitalização Prévia & $9(23,7)$ & $3(8,1)$ & $0,066^{*}$ & $2,16(0,78-5,90)$ \\
\hline Aleitamento Materno & $35(92,1)$ & $24(64,9)$ & $0,004 * *$ & $2,62(1,63-4,19)$ \\
\hline AME & $31(86,6)$ & $14(37,8)$ & $0,007 * *$ & $1,97(1,32-2,93)$ \\
\hline \multicolumn{5}{|l|}{ Socioeconômica e demográfica } \\
\hline Lixo a céu aberto & $5(13,2)$ & $4(10,8)$ & $0,754^{*}$ & $1,12(0,52-2,43)$ \\
\hline Água sem tratamento & $3(7,9)$ & $2(5,4)$ & $0,666^{*}$ & $1,25(0,42-3,7)$ \\
\hline Esgoto a céu aberto & $3(7,9)$ & $5(13,5)$ & $0,431 *$ & $0,76(0,42-1,38)$ \\
\hline \multicolumn{5}{|l|}{ Classe socioeconômica } \\
\hline$A-B$ & $7(18,4)$ & $11(29,7)$ & $0,826^{*}$ & $1,56(0,41-5,90)$ \\
\hline$c$ & $29(76,3)$ & $20(54,1)$ & $0,160 *$ & $2,37(0,70-8,04)$ \\
\hline D-E & $2(5,3)$ & $6(16,2)$ & $0,110^{*}$ & $1,16(0,88-4,90)$ \\
\hline \multicolumn{5}{|l|}{ Acompanhamento puericultura } \\
\hline Está em Acompanhamento & $35(92,1)$ & $32(86,5)$ & $0,431 *$ & $1,19(0,60-2,38)$ \\
\hline Número de Consultas Adequadas para Idade & $26(74,3)$ & $25(67,6)$ & $0,531 * *$ & $1,13(0,65-1,97)$ \\
\hline
\end{tabular}

*Teste Exato de Fisher; ** Teste Qui-quadrado; **RR: Razão de Risco e IC - Intervalo de Confiança de 95\%

Tabela 3. Avaliação geral da Atenção Primária conforme grupo intervenção e grupo controle

\begin{tabular}{l|cccc}
\hline Avaliação geral & Grupo intervenção $\mathrm{n}(\%)$ & Grupo controle $\mathrm{n}(\%)$ & $p$-value* & $\mathrm{RP}(\mathrm{IC}$ 95\%) \\
\hline$\geq 6,6$ (Boa orientação) & $23(60,5)$ & $18(48,6)$ & 0,302 & $1,27(0,79-2,02)$ \\
$\leq 6,6$ (Baixa orientação) & $15(39,5)$ & $19(51,4)$ & 1 \\
\hline
\end{tabular}

*Teste Qui-Quadrado; RP - Razão de Prevalência

Tabela 4. Avaliação dos atributos da Atenção Primária em Saúde estratificados nos dois grupos de lactentes analisados. Palmeira das Missões

\begin{tabular}{lccc}
\hline Avaliação por Atributo & $\begin{array}{c}\text { Grupo Intervenção Média (Desvio- } \\
\text { Padrão) }\end{array}$ & $\begin{array}{c}\text { Grupo Controle Média (Desvio- } \\
\text { Padrão) }\end{array}$ & $p$-value* \\
\hline Avaliação Geral $(\Sigma)$ & $6,979(1,2)$ & $6,094(1,8)$ & 0,016 \\
Acessibilidade de Primeiro Contato C & $5,874(1,2)$ & $5,589(1,3)$ & 0,333 \\
Longitudinalidade D & $7,538(1,1)$ & $7,059(1,9)$ & 0,190 \\
Integração de Cuidados E & $7,533(2,5)$ & $5,555(1,5)$ & 0,318 \\
Sistema de Informação F & $8,640(1,3)$ & $8,153(3,0)$ & 0,379 \\
Serviços Disponíveis G & $6,566(1,9)$ & $7,465(2,1)$ & 0,073 \\
Serviços Prestados H & $8,170(2,2)$ & $6,108(3,9)$ & 0,006 \\
Orientação Familiar I & $7,515(2,3)$ & $6,898(3,2)$ & 0,348 \\
Orientação Comunitária J & $6,389(2,9)$ & $6,979(2,8)$ & 0,436 \\
\hline
\end{tabular}

*Teste T

\section{Discussão}

No cenário do estudo, a avaliação geral da orientação à APS foi melhor avaliada naqueles participantes que possuem vínculo a ESF com as atividades de extensão universitárias, quando comparadas as demais ESF do município. Além disso, o atributo serviços prestados, corrobora com essa melhor avaliação, a medida que esse objetivo da atividade de extensão (prestação de serviços comunitários) está sendo alcançado.

A adesão ao aleitamento materno e aleitamento materno exclusivo foi maior no grupo intervenção.
Uma estratégia fundamental para adesão da amamentação, são as atividades educativas e de suporte materno durante o pré-natal e puericultura. Esse resultado demonstra que um dos objetivos da atividade extensionista, ou seja, os processos educativos em saúde foram atingidos por meio da consulta de puericultura. As atividades educativas de incentivo a amamentação realizadas durante as atividades de extensão, podem ter apoiado a família do lactante a aderir ao aleitamento materno exclusivo. ${ }^{(10)}$

O processo de amamentação é multifatorial, depende das condições biológicas e clínicas da puérpera 
e do lactente, empoderamento materno e familiar sobre a importância de amamentar bem como suporte social. ${ }^{(11)}$ Estratégias de educação em saúde, podem ser utilizadas para melhor adesão da amamentação do pré-natal e são estratégias que devem ser realizadas pela equipe de saúde, na APS. ${ }^{(10)}$ Por considerar a adesão a amamentação, uma prática multifatorial, entende-se que as inúmeras fontes educativas existentes (internet, apoio social, etc) precisam ser confiáveis e reportar práticas seguras de amamentação. É no momento da puericultura, que podem ser discutidas as experiências exitosas e dúvidas da nutriz, quanto às informações recebidas por outros meios, o que pode favorecer e aumentar taxas de adesão.

O grupo intervenção apresentou forte orientação da APS, com média de avaliação de 6,9, diferentemente do grupo controle que apresentou fraca orientação da APS. Essa diferença entre os grupos foi significativa, podendo ser relacionada as ações do projeto na direção de apontar melhoria da orientação da APS. O atributo que teve, significativamente, melhor avaliação no grupo intervenção foi a prestação de serviços.

Em estudo nacional, que avaliou a orientação da APS, utilizando o instrumento PCATOOL Brasil, versão para população de lactentes (menores de dois anos), em unidades de saúde apresentou escores menores, ${ }^{(12,13)}$ ao encontrado nos dois grupos analisados nesse estudo. Estudo nacional, com população pediátrica, quando comparados aos achados no presente estudo, aponta piores índices na orientação a APS, classificados assim como baixa orientação, nos serviços primários em saúde. Por isso, os melhores escores, apresentados no presente estudo podem estar associados as características locais de organização dos serviços bem como a presença da extensão universitária local. ${ }^{(14)}$

As melhores avaliações dos serviços de assistência à criança estão relacionadas à vinculação e ao relacionamento com os profissionais de saúde, ${ }^{(15)}$ dado este que justifica e corrobora com a melhor avaliação nas ESF com a intervenção, a medida que o projeto permite acolher e realizar vínculo dos participantes com a comunidade. No contexto pediátrico, estudo nacional corrobora com os achados a medida que destaca a acessibilidade como um dos atributos de maior fragilidade e limitante na APS. ${ }^{(15,16)}$ Esses achados orientam a formação acadêmica dentro do contexto da atenção primária e no Sistema Único de Saúde(SUS) e fortale- cem a manutenção de atividades extensionistas nesses contextos emergentes e necessários (fortalece formação e prestação de serviço para/no SUS).

Entende-se como limitação do estudo o pequeno número e a descrição de uma situação local (um município como cenário), a comparação entre grupos não equivalentes, a amostra por conveniência, mesmo que aleatória nos serviços de saúde, retratou apenas realidades de quem frequenta os serviços da APS do município.

\section{Conclusão}

No cenário onde foram desenvolvidas as atividades de extensão universitária, por meio das consultas de puericultura, houve uma melhor avaliação da orientação a APS sob perspectiva do escore geral e do atributo prestação de serviço, quando comparados serviços sem as atividades de extensão em puericultura. Ainda, as atividades do projeto não aumentaram significativamente o número de consultas de puericultura realizadas na população de lactentes, mas contribuiu aumentando a adesão de aleitamento materno e aleitamento materno exclusivo, naqueles com até seis meses de vida. Recomenda-se a continuidade e incentiva-se a comunidade acadêmica a realização das atividades extensionistas universitária no campo da saúde em especial na APS, na perspectiva da melhoria dos serviços e qualidade de vida e saúde da população. Ainda, são incipientes dados epidemiológicos e de avaliações dos serviços de atenção à saúde, na perspectiva de familiares dos lactentes, o que gera necessidade de maiores estudos de intervenção e avaliação.

\section{Contribuições}

Bartsch L, Loronha MF, Pioczkoski NP, Cavalheiro VS, Arrue AM e Jantsch LB contribuíram com a concepção do estudo, analise e interpretação dos dados, redação do artigo, revisão crítica relevante do conteúdo intelectual e aprovação da versão final a ser publicada.

\section{Referências}

1. Brasil. Ministério da Saúde. Secretaria de Atenção Básica. Manual do instrumento de avaliação da atenção primária à saúde: Primary Care Assessment Tool PCATool Brasil [Internet]. Brasília (DF): Ministério da Saúde; 2010 [citado 2019 jun 18]. Disponível em: http://bvsms.saude.gov.br/bvs/publicacoes/manual_avaliacao_pcatool_brasil.pdf 
2. Brito GV, Albuquerque IM, Ribeiro MA, Ponte EC, Moreira RM, Linhares MD. Consulta de puericultura na estratégia saúde da família: Percepção de enfermeiros. Rev APS. 2018;21(1):48-55.

3. Brasil. Ministério da Saúde. Secretaria de Atenção à Saúde. Cadernos de Atenção Básica: Saúde da Criança: Crescimento e Desenvolvimento [Internet]. Brasília (DF): Ministério da Saúde; 2014 [citado 2019 jun 18]. Disponível em https://bvsms.saude.gov.br/bvs/ publicacoes/saude_crianca_crescimento_desenvolvimento_1ed.pdf

4. Bahu LZ, Carbinatto MV. Extensão Universitária e ginástica para todos: Contribuições à formação profissional. Rev Conexões. 2016;14(3):46-70.

5. Fórum de Pró-Reitores das Instituições Públicas de Educação Superior Brasileiras. Política Nacional de Extensão Universitária [Internet]. Manaus (AM): Fórum de Pró-Reitores das Instituições Públicas de Educação Superior Brasileiras; 2012 [citado 2019 jun 18]. Disponível em https://proex.ufsc.br/files/2016/04/Pol\%C3\%ADtica-Nacional-deExtens\%C3\%A3oUniversit\%C3\%A1ria-e-book.pdf

6. Moraes SL, Tamaki R, Sobral AP, Santiago Júnior JF, Leão RD, Silva BG, Gomes JM. Impact of an extension experience in university education. Rev Cir Traumatol Buco-Maxilo-Fac. 2016;16(1):39-44.

7. Starfield B. Atenção Primária: equilíbrio entre necessidades de saúde, serviços e tecnologia. Brasilia (DF): Ministério da Saúde; 2002. 726p [citado 2019 jun 18]. Disponivel em http://www. dominiopublico.gov.br/pesquisa/DetalheObraForm.do?select_action=\&co_obra=14609

8. Coutinho SE, Reichert AP, Nogueira JA, Toso BR, Collet N. Health assessment: processual and structural dimension of child health in primary care. Saúde Debate. 2020;44(124):115-29.
9. Instituto Brasileiro de Geografia e Estatística (IBGE). Cidades e Estados 2019. Brasília (DF): Instituto Brasileiro de Geografia e Estatística; 2019 [citado 2020 jun 18]. Disponível em https://www.ibge.gov.br/cidades-e-estados/rs/palmeira-das-missoes.html

10. Brasil. Ministério da Saúde. Secretaria de Atenção Primária à Saúde. Departamento de Saúde da Família. Manual do Instrumento de Avaliação da Atenção Primária à Saúde: PCATool-Brasil - 2020 [Internet]. Brasília (DF): Ministério da Saúde; 2020 [citado 2020 jun 18]. Disponivel em: conasems.org.br/wp-content/uploads/2020/05/Pcatool_2020.pdf

11. Piro SS, Ahmed HM. Impacts of antenatal nursing interventions on mothers' breastfeeding self-efficacy: an experimental study. BMC Pregnancy Childbirth. 2020;20(1):19.

12. Moraes GG, Christoffel MM, Toso BR, Viera CS. Association between duration of exclusive breastfeeding and nursing mothers' self-efficacy for breastfeeding. Rev Esc Enferm da USP. 2021;55:e03702.

13. Silva GS, Alves CR. Evaluating the degree of implementation of primary health care attributes as an indicator of quality of care provided to children. Cad Saúde Pública. 2019;35(2):e00095418.

14. Samelli AG, Tomazelli GA, Almeida MH, Oliver FC, Melo SR, Avejonas DR. Avaliação do cuidado ao bebê de risco: comparação de modelos de atenção primária à saúde. Rev Saude Publica. 2019;53:98

15. Silva SA, Fracolli LA. Evaluating child care in the Family Health Strategy. Rev Bras Enferm. 2016;69(1):54-61.

16. Silva GS, Fernandes DRF, Alves CRL. Evaluation of primary child health care in Brazil: a systematic review of methods and results . Ciênc Saúde Colet. 2020;25(8):3185-200. 